\title{
Effect of a fixed-ratio (1:1:1) transfusion protocol versus laboratory-results-guided transfusion in patients with severe trauma: a randomized feasibility trial
}

\author{
Bartolomeu Nascimento MD MSc, Jeannie Callum MD, Homer Tien MD MSc, Gordon Rubenfeld MD MSc, \\ Ruxandra Pinto PhD, Yulia Lin MD, Sandro Rizoli MD PhD
}

\begin{abstract}
- Abstract
Background: Hemorrhage coupled with coagulopathy remains the leading cause of preventable in-hospital deaths among trauma patients. Use of a transfusion protocol with a predefined ratio of 1:1:1 (1 each of red blood cells [RBC], frozen plasma [FP] and platelets) has been associated with improved survival in retrospective studies in military and civilian settings, but such a protocol has its challenges and may increase the risk of respiratory complications. We conducted a randomized controlled trial to assess the feasibility of a 1:1:1 transfusion protocol and its effect on mortality and complications among patients with severe trauma.
\end{abstract}

Methods: We included 78 patients seen in a tertiary trauma centre between July 2009 and October 2011 who had hypotension and bleeding and were expected to need massive transfusion ( $\geq 10$ RBC units in $24 \mathrm{~h}$ ). We randomly assigned them to either the fixed-ratio $(1: 1: 1)$ transfusion protocol $(n=40)$ or to a laboratory-results-guided transfusion protocol (control; $n=38$ ). The primary outcome, feasi- fixed-ratio (1:1:1) transfusion strategy is a resuscitation strategy for trauma patients that promotes the transfusion of red blood cells (RBC), plasma and platelets (PLT) at a 1:1:1 ratio while minimizing crystalloid infusion. ${ }^{1}$ This balanced transfusion strategy aims to correct both the early coagulopathy of trauma and the volume status of patients in hemorrhagic shock, thus targeting preventable hemorrhage-related deaths. ${ }^{2,3}$ Retrospective studies of the 1:1:1 transfusion protocol reported marked reductions in mortality based on retrospectively calculated ratios of plasma:PLT:RBC. ${ }^{4-6}$ Methodologic limitations, particularly survivorship bias (where higher mortality was associated with low ratios of plasma and PLT to RBC in unsalvageable patients who died before 1:1:1 transfusion could be achieved), preclude any definitive con- bility, was assessed in terms of blood product ratios and plasma wastage. Safety was measured based on 28-day mortality and survival free of acute respiratory distress syndrome.

Results: Overall, a transfusion ratio of 1:1:1 was achieved in $57 \%$ (21/37) of patients in the fixed-ratio group, as compared with $6 \%(2 / 32)$ in the control group. A ratio of 1:1 (RBC:FP) was achieved in $73 \%$ (27/37) in the fixed-ratio group and $22 \%(7 / 32)$ in the control group. Plasma wastage was higher with the intervention protocol (22\% [86/390] of FP units v. $10 \%$ [30/289] in the control group). The 28-day mortality and number of days free of acute respiratory distress syndrome were statistically similar between the groups.

Interpretation: The fixed-ratio transfusion protocol was feasible in our study, but it was associated with increased plasma wastage. Larger randomized trials are needed to evaluate the efficacy of such a protocol in trauma care. Trial registration: ClinicalTrials.gov, no. NCT00945542
Competing interests: See end of article.

This article has been peer reviewed.

Correspondence to:

Sandro Rizoli,

rizolis@smh.ca

CMAJ 2013. DOI:10.1503 /cmaj.121986 clusion on the potential benefit of a 1:1:1 transfusion strategy in terms of efficacy and safety. ${ }^{7-10}$

The 1:1:1 transfusion strategy has been widely adopted by trauma centres worldwide ${ }^{11,12}$ and is being increasingly used in prehospital care and in the care of patients without traumatic injuries..$^{13-15}$ Widespread adoption of the strategy has significant resource and safety implications. Its full implementation requires access to thawed type $A B$ plasma, which is chronically in short supply. ${ }^{16}$ In addition, because of the difficulty in predicting the need for massive transfusion (commonly defined as $\geq 10 \mathrm{RBC}$ units in $24 \mathrm{~h}$ ), the 1:1:1 transfusion protocol may lead to unnecessary exposure to blood components and an increased risk of acute respiratory distress syndrome, sepsis and multiple organ dysfunction. ${ }^{17}$

We conducted a pilot randomized controlled 
trial comparing a 1:1:1 transfusion strategy with the standard of care at our institution (laboratoryresults-guided transfusion; laboratory results are available for transfusion decisions throughout resuscitation) in trauma patients predicted to need massive transfusion. Our primary objective was to assess the feasibility and safety of the fixedratio protocol in patients with severe trauma.

\section{Methods}

\section{Study design and participants}

The study design, methods and preliminary feasibility data have been previously reported. ${ }^{18}$ In brief, the study was a single-centre, unblinded, randomized controlled trial with a 2-arm parallel-group design that enrolled patients between July 2009 and October 2011.

Patients with traumatic injuries were eligible if they were 16-90 years old; had bleeding and were expected to require massive transfusion (either anticipated need for 4 units of RBC within the next $2 \mathrm{~h}$ or $\geq 10$ units of RBC in $24 \mathrm{~h}$, or required uncrossmatched $\mathrm{RBC}$ ); and had an episode of systolic blood pressure $\leq 90 \mathrm{~mm} \mathrm{Hg}$.

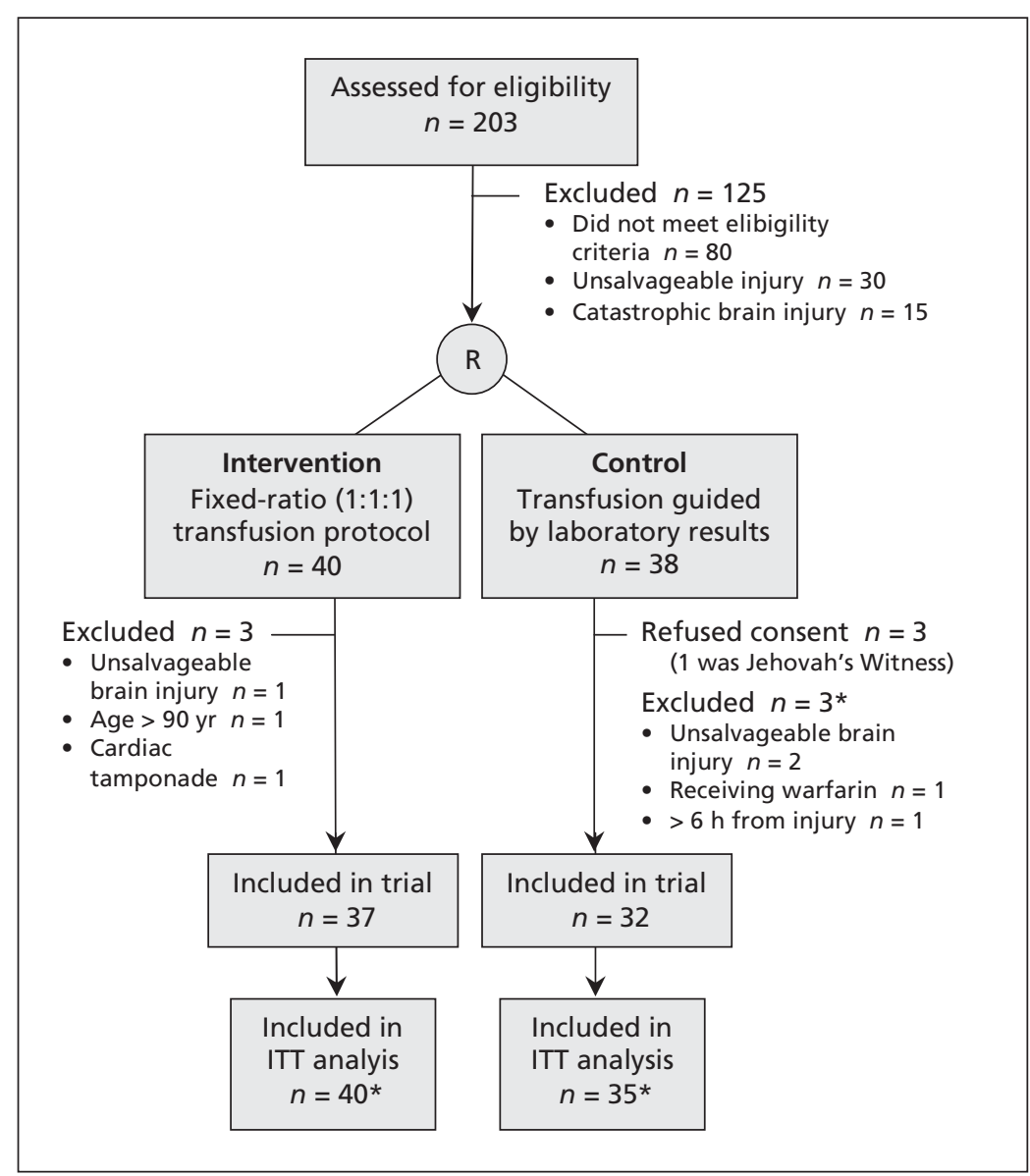

Figure 1: Flow of patients through the trial. *The intention-to-treat (ITT) analysis of all-cause 28-day mortality included the patients who were excluded after randomization.
We excluded patients if they arrived more than 6 hours after injury; received more than 2 units of $\mathrm{RBC}$ before arrival; had a severe brain injury (defined as any of a score of 3 on the Glasgow Coma Scale owing to brain injury; need of immediate neurosurgery; focal signs such as anisocoria; or computed tomography $[\mathrm{CT}]$ evidence of intracranial bleeding with mass effect); had a catastrophic brain injury (defined as transcranial gunshot wound, open skull fracture with exposure or loss of brain tissue, or expert medical opinion based on initial clinical or CT findings); had shock unrelated to hemorrhage (i.e., cardiogenic, septic, neurogenic or obstructive [cardiac tamponade, tension pneumothorax or massive pulmonary emboli]); had an underlying hereditary or acquired coagulopathy; or were moribund and unlikely to survive more than a few hours.

Written informed consent was obtained from all patients or substitute decision-makers for participation in the study. For patients unable to consent and without substitute decision-makers, the consent was delayed in accordance with established criteria. ${ }^{19}$

The study design was approved by the Sunnybrook Research Ethics Board.

\section{Randomization and masking}

Research coordinators were responsible for reviewing whether patients met the inclusion and exclusion criteria. Eligible patients were randomly assigned to the study groups by the hospital's blood bank technologists. A computerized random-number generator was used to generate sequences of random numbers. Allocation was concealed within sealed opaque envelopes in the blood bank. Allocation sequence was derived from blocks of 4 for the control and intervention groups, and randomization was stratified by type of trauma (blunt or penetrating) to assure balanced groups.

\section{Study protocol}

If assigned to the intervention group, patients received transfusions of RBCs, frozen plasma (FP) and PLT at a 1:1:1 ratio. Pre-thawed plasma was not available for this study; FP was thawed on demand. Therefore, RBC units were transfused as clinically indicated until randomized blood products were available in the 1:1:1 ratio. Four FP units, 1 pool of PLT derived from the buffy coat (from 4 individual donor units) and $4 \mathrm{RBC}$ units were issued as a set. Once the first set of products was issued, the blood bank technologists immediately prepared another identical set of blood components. In the fixed-ratio group, laboratory testing was performed at the discretion of the attending physician.

Patients assigned to the control group were managed according to the institution's usual proto- 
col for massive transfusion..$^{18}$ In this protocol, blood work (including complete blood count, international normalized ratio [INR], partial thromboplastin time and fibrinogen) is recommended at least every 2 hours for the duration of the protocol phase in order to guide transfusion decisions. Transfusions of RBC units were given if the hemoglobin level dropped to $70 \mathrm{~g} / \mathrm{L}$ or lower. Frozen plasma was transfused in doses of 3-4 units to maintain an INR of less than 1.8. Platelet transfusions were given to patients 1 pool (4 units) at a time if the PLT count was less than $50 \times 10^{9} / \mathrm{L}$.

The study protocols were followed for a maximum of 12 hours, unless they were stopped earlier if the attending physician or surgeon felt that hemostasis was achieved.

\section{Outcome measures}

The primary outcome was feasibility, as measured by the proportion of patients in the fixed-ratio group who received appropriate blood products in the predefined (1:1:1) ratio. Based on results of retrospective studies, ${ }^{3,46}$ we determined that patients received the predefined ratio if, for each FP unit, they received between 0.8 and $1.3 \mathrm{RBC}$ units and at least 0.8 pooled PLT units. We also measured the difference between the 2 study groups with respect to number of units and time to transfusion of blood products received, as well as the transfusion ratios. We measured RBC:FP ratios separately to assess the feasibility of rapidly thawing FP units and issuing them at a 1:1 ratio with $\mathrm{RBC}$ units.

We measured safety in terms of the 28-day mortality (all-cause mortality and rate of death by exsanguination); incidence of any degree of acute respiratory distress syndrome (based on the Berlin consensus definition for acute respiratory distress syndrome ${ }^{20}$ ), measured as days free of acute respiratory distress syndrome to account for mortality bias, ${ }^{21}$ and transfusion-related complications, identified using the Canadian Transfusion Transmitted Injuries Surveillance System criteria. ${ }^{22}$ To measure the effect of the 2 strategies on clinical practice and adherence to the control protocol, we evaluated the differences in blood product wastage between groups and frequency of blood work performed during massive transfusion protocol (expected to be at least every $2 \mathrm{~h}$ for patients in the control group). ${ }^{10}$

\section{Statistical analysis}

Because this was a feasibility study, no calculation of sample size was required. Based on data in our institution's trauma registry, the number of patients needing massive transfusion per year would be 40 patients, with $10 \%$ of eligible patients missed. Thus, our target study population was 36 patients in each arm over a 2-year feasibility period. Because of the lack of previ- ous prospective experience with ratio-based transfusion protocols, no clinically important difference between study groups was set a priori.

Summary data for continuous variables are presented as means and standard deviations, or medians and interquartile ranges depending on the distribution. Discrete variables are summarized as frequency and percentages. We assessed differences in binary feasibility outcomes and mortality outcomes using the $\chi^{2}$ or Fisher exact test. Allcause 28-day mortality was analyzed by the intention-to-treat principle. We used the Wilcoxon ranksum test to analyze ratios of blood and blood products; differences in RBC:FP and FP:PLT ratios were reported separately. We calculated the $95 \%$ confidence interval (CI) for the median difference in continuous variables between the 2 groups using the bootstrap technique based on 10000 simulations. For categorical clinical outcomes, we calculated relative risks (RRs) and 95\% CIs using the bootstrap technique based on 10000 simulations. We analyzed overall survival and survival free of acute respiratory distress syndrome as the time to first event (death or acute respiratory syndrome) using log-rank tests; the data are graphically presented using Kaplan-Meier curves. In addition to the statistical tests used for the binary and continu-

Table 1: Baseline characteristics of 69 trauma patients expected to require massive transfusion, * by allocation to the fixed-ratio $(1: 1: 1)$ transfusion protocol or the laboratory-results-guided transfusion protocol (control)

\begin{tabular}{|c|c|c|}
\hline Characteristic & $\begin{array}{l}\text { Fixed-ratio group } \\
\qquad n=37\end{array}$ & $\begin{array}{c}\text { Control group } \\
n=32\end{array}$ \\
\hline Age, yr, median (IQR) & $41(23-58)$ & $34(25-40)$ \\
\hline Sex, male, no. (\%) & $24(65)$ & $23(72)$ \\
\hline Penetrating trauma, no. (\%) & $13(35)$ & $12(38)$ \\
\hline Transferred from other hospital, no. (\%) & $7(19)$ & $5(16)$ \\
\hline $\begin{array}{l}\text { Time from injury to hospital, min, } \\
\text { median (IQR) }\end{array}$ & $46(30-59)$ & $45(30-67)$ \\
\hline Pre-hospital fluid, $\mathrm{mL}$, median (IQR) & $300(0-1500)$ & $625(0-1012)$ \\
\hline Injury severity score, mean \pm SD & $35 \pm 13$ & $35 \pm 13$ \\
\hline $\begin{array}{l}\text { Score }>3 \text { for head injury on } \\
\text { Abbreviated Injury Scale, }+ \text { no. (\%) }\end{array}$ & $14(38)$ & $11(34)$ \\
\hline Glasgow Coma Scale score, median (IQR) & $14(3-15)$ & $13(6-15)$ \\
\hline $\begin{array}{l}\text { Systolic blood pressure, } \mathrm{mm} \mathrm{Hg} \text {, } \\
\text { median (IQR) }\end{array}$ & $81(70-100)$ & $80(73-88)$ \\
\hline Temperature, ${ }^{\circ} \mathrm{C}$, mean $\pm \mathrm{SD}$ & $35 \pm 1.1$ & $35 \pm 1.1$ \\
\hline $\mathrm{pH}$, mean $\pm \mathrm{SD}$ & $7.13 \pm 0.2$ & $7.18 \pm 0.1$ \\
\hline INR, median (IQR) & $1.2(1.1-1.5)$ & $1.4(1.2-1.7)$ \\
\hline Fibrinogen, $g / L$, mean $\pm S D$ & $1.5 \pm 0.8$ & $1.2 \pm 0.6$ \\
\hline Platelet count, $\times 10^{9} / \mathrm{L}$, median (IQR) & $201(131-252)$ & $192(131-243)$ \\
\hline Hemoglobin, g/L, median (IQR) & $99(78-127)$ & $90(79-112)$ \\
\hline \multicolumn{3}{|c|}{$\begin{array}{l}\text { Note: INR }=\text { international normalized ratio, IQR }=\text { interquartile range, } S D=\text { standard deviation. } \\
\text { *Massive transfusion }=\geq 10 \text { units of red blood cells in } 24 \text { hours. } \\
\text { †Abbreviated Injury Scale values }>3 \text { denote severe head injury. }\end{array}$} \\
\hline
\end{tabular}


ous outcomes, we used the Student $t$ test to compare means of other secondary outcomes (see Appendix 1 for details, at www.cmaj.ca/lookup /suppl/doi:10.1503/cmaj.121986/-/DC1). No imputation was performed for missing data.

An independent data safety and monitoring board unaware of the group allocations closely monitored events related to transfusion complications, particularly acute lung injury and acute respiratory distress syndrome, for perceived discrepancies between the groups. ${ }^{18}$ The committee deliberated on the need to exclude patients who were mistakenly recruited and randomly assigned to a study arm. Because of the narrow window available to recruit critically injured patients in our study, complete information on eligibility was not always available at the time of recruitment. Therefore, some patients were found to meet exclusion criteria after enrolment and randomization. Furthermore, the committee advised on the "per-protocol" analysis of the transfusion data, because these ineligible patients were excluded after randomization.

Data were analyzed using IBM SPSS Statistics version 20 (IBM Corporation), SAS version 9.3 (SAS Institute Inc.) and R version 2.15.0 for Windows. All tests were 2 -sided, and $p$ values less than 0.05 were considered statistically significant.

\section{Results}

During the study period, we assessed 203 patients for eligibility, of whom 78 were randomly assigned to either the fixed-ratio $(n=40)$ or control group $(n=38)$. Nine of the 78 patients did not complete the study after randomization: 6 (3 in each group) were mistakenly enrolled owing to the narrow window for recruitment and were later found to meet the exclusion criteria, and 3 patients (control group) refused consent after randomization (see Appendix 1 for details). A total of 69 patients (37 in fixed-ratio and 32 in control group) were included in the analysis (Figure 1).

The 2 groups were balanced with respect to baseline characteristics (Table 1; for information on co-interventions, see Appendix 1). In both groups, the transfusion protocol remained activated for a median duration of 5 hours.

Table 2: Transfusion data and plasma wastage during study protocols

\begin{tabular}{|c|c|c|c|c|}
\hline Variable & $\begin{array}{l}\text { Fixed-ratio group } \\
\qquad n=37\end{array}$ & $\begin{array}{l}\text { Control group } \\
\quad n=32\end{array}$ & Difference $(95 \% \mathrm{Cl})$ * & $p$ valuet \\
\hline \multicolumn{5}{|l|}{ Transfusion data } \\
\hline $\begin{array}{l}\text { Ratio of RBC:FP:PLT achieved, } \neq \\
\text { median (IQR) }\end{array}$ & $\begin{array}{c}1: 1: 1 \\
\text { (1 to } 1.3: 1: 0.8 \text { to } 1.3 \text { ) }\end{array}$ & $\begin{array}{c}1.7: 1: 0.8 \\
\text { (1.2 to } 2.3: 1: 0.5 \text { to } 1.3 \text { ) }\end{array}$ & $\begin{array}{l}\text { RBC: }-0.7(-1.1 \text { to }-0.3) \\
\text { PLT: } \quad 0.2(-0.3 \text { to } 0.35)\end{array}$ & $\begin{array}{l}\text { RBC: }<0.01 \\
\text { PLT: } 0.3\end{array}$ \\
\hline Received 1:1:1 ratio, no. (\%) & $21(57)$ & $2(6)$ & 51 (32 to 68$)$ & $<0.01$ \\
\hline $\begin{array}{l}\text { Received only RBC:FP at 1:1 ratio, } \\
\text { no. (\%) }\end{array}$ & $27(73)$ & $7(22)$ & 51 (31 to 71$)$ & $<0.01$ \\
\hline $\begin{array}{l}\text { Total no. of RBC:FP:PLT units per } \\
\text { patient, } \S \text { median (IQR) }\end{array}$ & $\begin{array}{c}7: 6: 8 \\
\text { (6 to } 10: 4 \text { to } 8: 4 \text { to } 8 \text { ) }\end{array}$ & $\begin{array}{c}7: 4: 4 \\
\text { (6 to } 14: 3 \text { to } 8: 0 \text { to } 8 \text { ) }\end{array}$ & $\begin{array}{l}\text { RBC: } 0 \text { ( }-5 \text { to } 2.5) \\
\text { FP: } 2 \text { (0 to } 4) \\
\text { PLT: } 4 \text { ( }-3 \text { to } 6)\end{array}$ & $\begin{array}{ll}\text { RBC: } & 0.6 \\
\text { FP: } & 0.07 \\
\text { PLT: } & 0.1\end{array}$ \\
\hline Received FP transfusion, no. (\%) & $36(97)$ & $26(81)$ & 16.0 (1.5 to 30.5$)$ & 0.04 \\
\hline Received PLT transfusion, no. (\%) & $34(92)$ & $21(66)$ & 26.3 (7.6 to 44.9$)$ & 0.01 \\
\hline $\begin{array}{l}\text { Received massive transfusion } \\
(\geq 10 \text { RBC units in } 24 \mathrm{~h}) \text {, no. (\%) }\end{array}$ & $15(41)$ & $15(47)$ & $-6.3(-29.8$ to 17.1$)$ & 0.6 \\
\hline Time to first RBC, I min, median (IQR) & 25.5 (14 to 48.5$)$ & 32.5 (13 to 70.5$)$ & $-7.0(-23.5$ to 13.5$)$ & 0.8 \\
\hline Time to first FP, 1 min, median (IQR) & 89 (65 to 150$)$ & 113 (81 to 165$)$ & -24 (-60 to 9$)$ & 0.05 \\
\hline $\begin{array}{l}\text { Time from first RBC to first FP, min, } \\
\text { median (IQR) }\end{array}$ & 60 (40 to 77 ) & 78 (49 to 112 ) & $-19(-45$ to -1$)$ & 0.05 \\
\hline \multicolumn{5}{|l|}{ Plasma wastage } \\
\hline Total FP units thawed, no. & 390 & 289 & NA & NA \\
\hline FP units wasted, no. (\%) & $86(22)$ & $30(10)$ & NA & NA \\
\hline \multicolumn{5}{|c|}{$\begin{array}{l}\text { Note: } \mathrm{Cl}=\text { confidence interval, } \mathrm{FP}=\text { frozen plasma, IQR }=\text { interquartile range, } \mathrm{NA}=\text { not applicable, } \mathrm{PLT}=\text { platelets, } \mathrm{RBC}=\text { red blood cells, } \mathrm{SD}=\mathrm{standard} \text { deviation. } \\
\text { *For continuous data, estimation of the } 95 \% \mathrm{Cl} \text { for the median difference between the } 2 \text { groups was calculated using the bootstrap technique based on } 10000 \\
\text { simulations. } \\
+\chi^{2} \text { test or Fisher exact test to compare proportions; Wilcoxon rank-sum test to compare distributions; } p \text { values for differences in RBC, FP and PLT utilization } \\
\text { between study groups are reported separately. } \\
\text { fRatios of RBC:FP and FP:PLT calculated to } 1 \mathrm{FP} \text { unit. One patient in the fixed-ratio group and } 4 \text { in the control group did not receive any FP; } p \text { values for the } \\
\text { differences in RBC:FP and FP:PLT ratios between study groups are reported separately. } \\
\text { \$Absolute number of units of RBC, FP and PLT transfused per patient during study protocols. } \\
\text { qTime from hospital arrival to transfusion of first unit of RBC or FP; the time was not recorded for } 1 \text { patient in the fixed-ratio group. }\end{array}$} \\
\hline
\end{tabular}




\section{Feasibility outcomes}

At protocol termination, a transfusion ratio of 1:1:1 was achieved in 57\% (21/37) of the patients in the fixed-ratio group, as compared with $6 \%$ $(2 / 32)$ in the control group (absolute difference $51 \%, 95 \%$ CI $32 \%$ to $68 \%$ ). A ratio of $1: 1$ (RBC:FP) was achieved in $73 \%(27 / 37)$ in the fixed-ratio group and $22 \%(7 / 32)$ in the control group (absolute difference $51 \%, 95 \%$ CI $31 \%$ to $71 \%)$. The median RBC:FP:PLT ratios were higher in the control group than in the fixed-ratio group (1.7:1:0.8 v. 1.0:1:1.0) (Table 2).

Transfusion data, including time to RBC and plasma transfusions, and plasma wastage are shown in Table 2. The proportion of patients who received uncrossmatched RBC units did not differ significantly between the 2 groups ( $97 \%$ [36/37] in the fixed-ratio group v. $88 \%$ [28/32] in the control group; $p=0.2$ ). The number of units of thawed plasma wasted per study group was increased in the fixed-ratio group (Table 2).

During the laboratory-guided protocol, laboratory tests were performed at least every 2 hours in the vast majority of patients for hemoglobin con- centration, platelet count and international normalized ratio, but in less than 50\% for fibrinogen and partial thromboplastin time (Table 3).

\section{Safety outcomes}

In the intention-to-treat analysis, the all-cause 28day mortality was $32 \%(13 / 40)$ in the fixed-ratio group, as compared with $14 \%$ (5/35) in the control group (RR 2.27, 95\% CI 0.98 to 9.63). Additional mortality outcomes, coagulation competence (correction of the coagulopathy, defined as correction of the conventional laboratory tests by protocol cessation), bleeding-free hours, ventilator-free days, and lengths of stay in the intensive care unit and hospital are provided in Table 4 and Appendix 1.

Event-free survival was achieved by $54 \%$ (20/37) of the patients in the fixed-ratio group, as compared with 78\% (25/32) in the control group ( $p=0.053, \log$-rank test). The Kaplan-Meier survival curves and log-rank test for event-free survival are depicted in Figure 2. No transfusionrelated acute lung injury or major transfusion reactions were observed in either group.

Table 3: Compliance with target coagulation monitoring at least every 2 hours*

\begin{tabular}{|lccc|} 
& \multicolumn{2}{c}{ Group; no. (\%) of patients } & \\
\cline { 2 - 3 } Test & $\begin{array}{c}\text { Fixed-ratio group } \\
n=37\end{array}$ & $\begin{array}{c}\text { Control group } \\
n=32\end{array}$ & $p$ valuet \\
\hline International normalized ratio & $27(73)$ & $24(75)$ & 0.8 \\
\hline Fibrinogen & $13(35)$ & $12(38)$ & 0.8 \\
\hline Partial thromboplastin time & $13(35)$ & $14(44)$ & 0.6 \\
\hline Platelet count & $30(81)$ & $29(91)$ & 0.4 \\
\hline Hemoglobin & $30(81)$ & $29(91)$ & 0.4 \\
\hline $\begin{array}{l}* \text { *In the fixed-ratio group, laboratory testing at least every } 2 \text { hours was not part of the study protocol and was performed at the } \\
\text { discretion of the attending physician. } \\
+\chi^{2} \text { test. }\end{array}$ & & \\
\hline
\end{tabular}

Table 4: Mortality outcomes

\begin{tabular}{|c|c|c|c|c|}
\hline \multirow[b]{2}{*}{ Variable } & \multicolumn{2}{|c|}{ Group; $n / N(\%)$} & \multirow[b]{2}{*}{$\begin{array}{l}\text { Relative risk } \\
\quad(95 \% \mathrm{Cl})\end{array}$} & \multirow[b]{2}{*}{$\begin{array}{l}\text { Difference } \\
(95 \% \mathrm{Cl})\end{array}$} \\
\hline & $\begin{array}{l}\text { Fixed-ratio group } \\
\qquad n=37\end{array}$ & $\begin{array}{c}\text { Control group } \\
\quad n=32\end{array}$ & & \\
\hline All-cause 28-day mortality in ITT analysis* & $13 / 40(32.5)$ & $5 / 35(14.3)$ & 2.27 (0.98 to 9.63$)$ & $18.2(-0.4$ to 36.8$)$ \\
\hline All-cause 28-day mortality per protocol & $11 / 37(29.7)$ & $3 / 32 \quad(9.4)$ & $3.17(1.15$ to 18.24$) \ddagger$ & 20.3 (2.5 to 38.2$)$ \\
\hline Death from exsanguinationt & $8 / 37(21.6)$ & $3 / 32 \quad(9.4)$ & $2.30(0.74$ to 13.03$)$ & $12.2(-4.4$ to 28.9$)$ \\
\hline $\begin{array}{l}\text { Neurologic death (traumatic brain } \\
\text { injury/withdrawal of care) }\end{array}$ & $2 / 37 \quad(5.4)$ & $0 / 32$ & NA & $5.4(-1.8$ to 12.7$)$ \\
\hline Death from multiple organ failure & $1 / 37 \quad(2.7)$ & $0 / 32$ & NA & $2.7(-2.5$ to 7.9$)$ \\
\hline \multicolumn{5}{|c|}{$\begin{array}{l}\text { Note: } \mathrm{Cl}=\text { confidence interval, IQR }=\text { interquartile range, ITT }=\text { intention-to-treat, } \mathrm{NA}=\text { not applicable. } \\
\text { *For the ITT analysis, data were included for } 40 \text { patients in the fixed-ratio group and } 35 \text { patients in the control group (see Figure } 1) \text {. } \\
\text { tMedian time of occurrence after arrival to hospital was } 2.8 \text { hours }(\mathrm{IQR} 1.7-14) \text { in the fixed-ratio group and } 4.4 \text { hours (IQR } 1.7-14) \text { in the control group. } \\
\neq 95 \% \mathrm{Cl} \text { generated by bootstrap technique with } 10000 \text { simulations; a continuity correction of } 0.5 \text { was used when } 0 \text { event was present in the control group in any } \\
\text { of the simulations. }\end{array}$} \\
\hline
\end{tabular}




\section{Interpretation}

In our study, both the 1:1:1 RBC:FP:PLT transfusion ratio and the 1:1 RBC:FP ratio were achieved in a significantly higher proportion of patients in the fixed-ratio group than in the control group. These findings suggest that a fixedratio transfusion protocol is feasible, but it was associated with increased plasma wastage (about 2 units per patient). The laboratoryresults-guided transfusion protocol has its challenges because of the long turnaround times for the test results. ${ }^{18}$

Despite the significant difference between the study groups in the proportion of patients receiving transfusions at the predefined ratio, a higher compliance with fixed-ratio transfusion may have been reached had pre-thawed plasma been immediately available. We did not have pre-thawed plasma available because of low utilization and restrictions on reissuing of thawed plasma within 24 hours. Frozen plasma is thawed in a water bath over 20 minutes. Its labelling and transport to the clinical setting added to the total turnaround time for FP transfusion. The median delay between the first RBC transfusion (which in most cases established eligibility for the trial and triggered the initial call to the research team) and the first plasma transfusion was 60 minutes in the fixed-ratio arm. A recent study on blood product ratios reported similar delays in plasma transfusion. ${ }^{7}$ Of note, 3 patients (8\%) in the fixed-ratio group in our study had their pooled PLT units returned by the medical team after judging its transfusion as inappropriate despite the protocol.

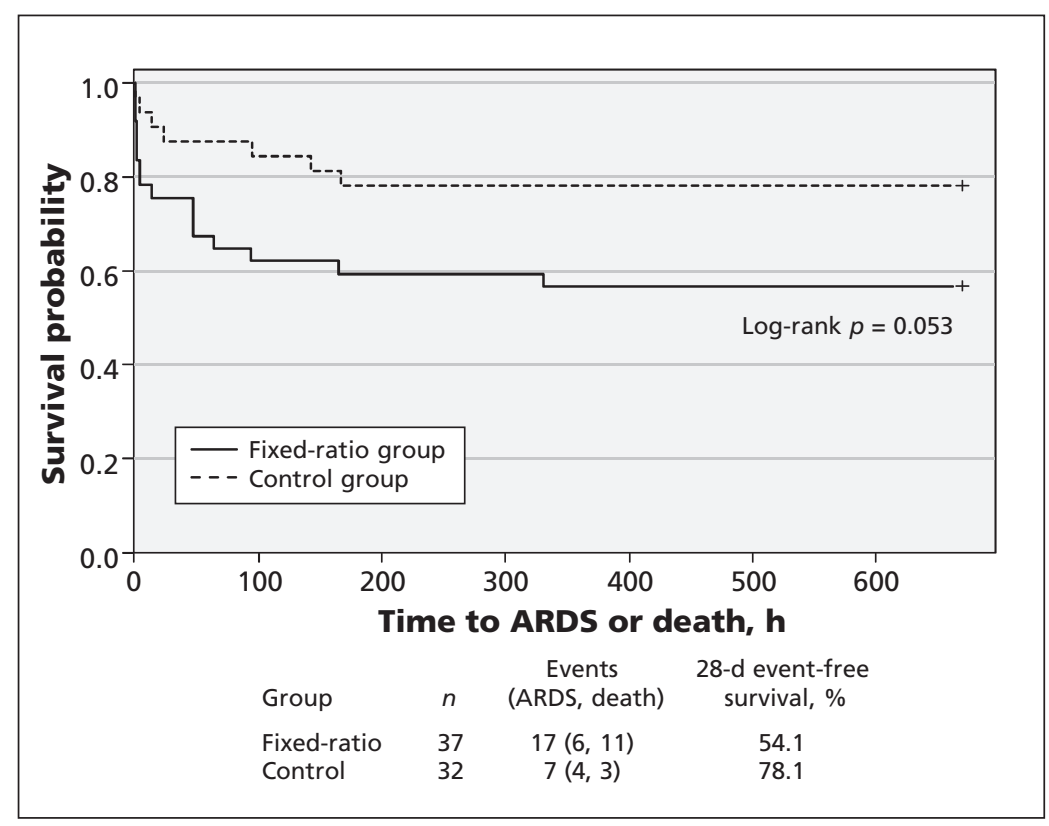

Figure 2: Kaplan-Meier curves for event-free survival (free of acute respiratory distress syndrome [ARDS] or death) within 28 days after enrolment.
Another patient in the fixed-ratio arm died early (within $1.1 \mathrm{~h}$ after arrival), which precluded FP and PLT transfusion.

In Canada, the recently revised Canadian Standards Association standard Z902-10 extended the use of thawed plasma from 24 hours to 5 days. ${ }^{23}$ However, the availability of thawed plasma at all times requires plasma from type $\mathrm{AB}$ donors (universal donors lacking anti-A and anti-B antibodies), which represent $4 \%$ of the donor population. ${ }^{10}$ Recently, as preventive measures to reduce the risk of transfusion-related acute lung injury, male-predominant plasma policies were implemented in most countries. In the United States, more than $40 \%$ of type $\mathrm{AB}$ plasma is from female donors, which is associated with an increased risk of transfusion-related acute lung injury. ${ }^{24} \mathrm{We}$ recorded an increase in plasma wastage with fixed-ratio transfusion, despite only using it in 37 patients without pre-thawed plasma. Previous studies on implementation of formula-driven care did not report wastage rates. The potential impact of widespread adoption of 1:1:1 transfusion protocol on plasma wastage and risk of transfusionrelated acute lung injury needs to be explored.

\section{Limitations}

Our trial was conducted in a setting where RBC units were readily available but not pre-thawed units of plasma, which represents an important limitation for the accomplishment of 1:1:1 transfusion. However, our system may be considered the current standard of practice in most trauma centres worldwide; rendering our findings generalizable. Also, survivorship bias, where unsalvageable patients die before $1: 1: 1$ transfusion ratio can be achieved, constitutes an issue inherent to our study population.

Although standard coagulation tests were used in a large proportion of patients in the control group, our study design did not allow for assessing the use of these results for transfusion decisions.

Random allocation of unstable trauma patients was challenging because of the narrow window for deciding on their eligibility. In future studies, eligibility could be determined in a 2-step process, with exclusion criteria being reviewed after the inclusion criteria are determined, but before randomization occurs.

In our small feasibility trial, clinical outcomes were presented as safety data only; thus, they should be interpreted with caution. ${ }^{25} \mathrm{~A}$ larger trial (the Pragmatic, Randomized Optimal Platelet and Plasma Ratios [PROPPR] trial), powered to evaluate the efficacy and safety of ratio-based transfusion strategies has begun (ClinicalTrials.gov, no. NCT01545232) and may clarify the role of a 1:1:1 transfusion strategy. 


\section{Conclusion}

Findings from our randomized controlled trial showed that implementation of a fixed-ratio (1:1:1) transfusion protocol was feasible among patients with severe trauma. The full and widespread implementation of such a protocol will challenge blood suppliers because of the increased demand (and wastage) of plasma. Larger clinical trials are warranted to definitively evaluate the efficacy and safety of transfusion at a 1:1:1 ratio.

\section{References}

1. Hess JR, Holcomb JB, Hoyt DB. Damage control resuscitation: the need for specific blood products to treat the coagulopathy of trauma. Transfusion 2006;46:685-6.

2. Holcomb JB, Jenkins D, Rhee P, et al. Damage control resuscitation: directly addressing the early coagulopathy of trauma. J Trauma 2007;62:307-10.

3. Zink KA, Sambasivan CN, Holcomb JB, et al. A high ratio of plasma and platelets to packed red blood cells in the first 6 hours of massive transfusion improves outcomes in a large multicenter study. Am J Surg 2009;197:565-70.

4. Borgman MA, Spinella PC, Perkins JG, et al. The ratio of blood products transfused affects mortality in patients receiving massive transfusions at a combat support hospital. J Trauma 2007; 63:805-13.

5. Cotton BA, Gunter OL, Isbell J, et al. Damage control hematology: the impact of a trauma exsanguination protocol on survival and blood product utilization. J Trauma 2008;64:1177-82.

6. Maegele M, Lefering R, Paffrath T, et al. Red-blood-cell to plasma ratios transfused during massive transfusion are associated with mortality in severe multiple injury: a retrospective analysis from the Trauma Registry of the Deutsche Gesellschaf fur Unfallchirurgie. Vox Sang 2008:95:112-9.

7. Snyder CW, Weinberg JA, McGwin G Jr, et al. The relationship of blood product ratio to mortality: Survival benefit or survival bias? J Trauma 2009;66:358-62.

8. Ho AM, Dion PW, Yeung JH, et al. Prevalence of survivor bias in observational studies on fresh frozen plasma:erythrocyte ratios in trauma requiring massive transfusion. Anesthesiology 2012;116: 716-28.

9. Nascimento B, Callum J, Rubenfeld G, et al. Clinical review: fresh frozen plasma in massive bleedings - more questions than answers. Crit Care 2010;14:202.

10. Dzik WH, Blajchman MA, Fergusson D, et al. Clinical review: Canadian National Advisory Committee on Blood and Blood Products - massive transfusion consensus conference 2011: report of the panel. Crit Care 2011;15:242.

11. Young PP, Cotton BA, Goodnough LT. Massive transfusion protocols for patients with substantial hemorrhage. Transfus Med Rev 2011;25:293-303

12. Levi M, Fries D, Gombotz H, et al. Prevention and treatment of coagulopathy in patients receiving massive transfusions. Vox Sang 2011;101:154-74

13. Smoot D, Park M, Osborn J, et al. Pre-hospital thawed plasma: a preliminary report. Proceedings of the 41 st annual meeting of Western Trauma Association; 2011 Feb. 27-Mar. 4; Big Sky (MT). Paper 36:116.

14. Forcione DG, Alam HB, Kalva SP, et al. Case records of the Massachusetts General Hospital. Case 9-2009. An 81-year-old man with massive rectal bleeding. N Engl J Med 2009;360:1239-48.

15. Saule I, Hawkins N. Transfusion practice in major obstetric haemorrhage: lessons from trauma. Int J Obstet Anesth 2012;21: 79-83.

16. Petrides M, Stack G, Cooling L, et al. Carbohydrate blood group antigens and collections. In: Practical guide to transfusion medicine. 2nd ed. Bethesda (MD): AABB Press; 2007. p. 59-91.

17. Inaba K, Branco BC, Rhee P, et al. Impact of plasma transfusion in trauma patients who do not require massive transfusion. $\mathrm{J} \mathrm{Am}$ Coll Surg 2010;210:957-65.

18. Nascimento B, Rizoli S, Rubenfeld G, et al. Design and preliminary results of a pilot randomized controlled trial on a $1: 1: 1$ transfusion strategy: the trauma formula-driven versus laboratory-guided study. J Trauma 2011;71(Suppl 1):S418-26.

19. Tri-Council policy statement: ethical conduct for research involving humans [article 3.8]. Canadian Institutes of Health Research, Natural Sciences and Engineering Research Council of Canada, and Social Sciences and Humanities Research Coun- cil of Canada; 2010. Available: www.pre.ethics.gc.ca/pdf/eng /tcps2/TCPS_2_FINAL_Web.pdf (accessed 2012 June 12).

20. Ranieri VM, Rubenfeld GD, Thompson BT, et al.; ARDS Definition Task Force. Acute respiratory distress syndrome: the Berlin Definition. JAMA 2012;307:2526-33.

21. Bulger EM, Jurkovich GJ, Nathens AB, et al. Hypertonic resuscitation of hypovolemic shock after blunt trauma: a randomized controlled trial. Arch Surg 2008;143:139-48, discussion 149.

22. Transfusion Transmitted Injuries Surveillance System: user's manual, version 3.0. Ottawa (ON): Public Health Agency of Canada; 2007. Available: www.phac-aspc.gc.ca/hcai-iamss/tti-it /pdf/users_manual_07_e.pdf (accessed 2012 June 12).

23. Blood and blood components [Table 2]. Mississauga (ON): Canadian Standards Association; 2010. p. 98.

24. Eder AF, Herron RM Jr, Strupp A, et al. Effective reduction of transfusion-related acute lung injury risk with male-predominant plasma strategy in the American Red Cross (2006-2008). Transfusion 2010;50:1732-42.

25. Arnold DM, Burns KE, Adhikari NK, et al.; McMaster Critical Care Interest Group. The design and interpretation of pilot trials in clinical research in critical care. Crit Care Med 2009;37(Suppl 1): S69-74.

Competing interests: Bartolomeu Nascimento received the 2010 National Blood Foundation Grant of the American Association of Blood Banks for the conduct of research related to coagulopathy in trauma. Yulia Lin is a site investigator for a registry on the off-label use of recombinant factor VIIa that is funded by an unrestricted educational grant from NovoNordisk. Sandro Rizoli received a Canadian Institutes of Health Research investigator award in partnership with NovoNordisk, the manufacturer of recombinant factor VIIa; he was also a consultant for NovoNordisk in the past and is a member of the advisory board for CSL Behring, manufacturer of fibrinogen concentrate. No competing interests declared by the other authors.

Affiliations: Departments of General Surgery (Nascimento) and Trauma and Critical Care (Rubenfeld), Division of General Surgery (Tien), Trauma, Emergency and Critical Care Program (Pinto), Sunnybrook Health Sciences Centre, University of Toronto; Department of Laboratory Medicine and Pathobiology (Callum, Lin), University of Toronto; Department of Clinical Pathology (Callum, Lin), Sunnybrook Health Sciences Centre; Canadian Forces Health Services (Tien); Departments of Surgery and Critical Care Medicine (Rizoli), St. Michael's Hospital, University of Toronto, Toronto, Ont.

Contributors: Bartolomeu Nascimento and Sandro Rizoli had full access to all of the data and take responsibility for the integrity and accuracy of the data. Bartolomeu Nascimento, Sandro Rizoli, Jeannie Callum, Homer Tien, Gordon Rubenfeld and Yulia Lin contributed to the study concept and design. Bartolomeu Nascimento, Sandro Rizoli and Jeannie Callum supervised the study. Bartolomeu Nascimento, Sandro Rizoli, Jeannie Callum, Ruxandra Pinto and Gordon Rubenfeld analyzed and interpreted the data. Bartolomeu Nascimento and Ruxandra Pinto performed the statistical analysis. Bartolomeu Nascimento and Sandro Rizoli drafted the manuscript. All of the authors critically reviewed the manuscript for important intellectual content. All of the authors approved the final version of the manuscript submitted for publication.

Funding: This study was funded by grants from the Canadian Forces Health Services; Defense Research and Development Canada; and the National Blood Foundation, American Association of Blood Banks. The funding agencies had no participation or role in the study design, data collection, data analysis, data interpretation, writing of the manuscript or decision to submit the study findings for publication.

Acknowledgements: The authors acknowledge the fundamental support of the blood bank team for the conduct of this trial, as well as the trauma registry personnel for the invaluable help with the trauma registry data. They also acknowledge all of the research assistants, coordinators and manager of the Tory Regional Trauma Centre for their excellent help with data collection and entry. 